\title{
Erratum: Distinguishing Elastic Shear Deformation from Friction on the Surfaces of Molecular Crystals [Phys. Rev. Lett. 104, 086102 (2010)]
}

\author{
Vivek Kalihari, Greg Haugstad, and C. Daniel Frisbie \\ (Received 2 April 2010; published 23 April 2010)
}

We have discovered that the fitting in Fig. 3(a) of the published Letter is not correct because we used the reported elastic constants of anthracene from Ref. [23] which follows the Voight notation and employed them in an equation from Ref. [22] which follows non-Voight notation. Also, the $\mathbf{a}$ and $\mathbf{b}$ axes used in Ref. [23] are inverted with respect to our notation.

In Fig. 1 here, the filled triangles correspond to experimental transverse shear microscopy (TSM) data obtained on a pentacene single crystal and the solid line represents the corresponding fit using the TSM equation from our published Letter. The only difference between Fig. 3(a) and the Fig. 1 here is that in Fig. 3(a) the solid line represented the TSM plot for anthracene single crystal based on its elastic constants, whereas in Fig. 1 the solid line is a fit for the pentacene experimental data. The fit in Fig. 1 gives the following relative $a-b$ in-plane elastic constants for pentacene: $E_{1111} \sim$ $1.8 E_{2222}, E_{1212} \sim 0.2 E_{2222}, E_{1122} \sim 0.6 E_{2222}, E_{1112} \ll E_{2222}, E_{2212} \ll E_{2222}$, and $G$ has a negative value. The dashed line in Fig. 1 represents the modified TSM plot when the value of $E_{1212}$ is increased by $\sim 60 \%$ and all the other in-plane elastic constants are kept unchanged.

The correction does not change any fundamental conclusions of the Letter. It only changes the reported relative in-plane elastic constants for a pentacene single crystal based on the new fit.

We thank Dr. Marcello Campione (Università degli Studi di Milano Bicocca) for his questions which led to the discovery of our mistake.



FIG. 1 (color online). Plot of TSM signal versus scanning direction $(\theta)$. The filled triangles correspond to experimental TSM data and the solid line is a corresponding fit using the TSM equation. The dashed line represents the modified TSM plot when the value of $E_{1212}$ is increased by $\sim 60 \%$. 